\title{
Clinical effect of combined anterior and posterior approach surgery for cervical spondylotic myelopathy
}

\author{
JIANG Yan-zhou, FU Lian-chong, MU Yun-shan \\ People's Hospital of Hanting District, Weifang 261100, China
}

Abstract: Objective: To evaluate the clinical efficacy of combined anterior and posterior approach surgery for patients with spinal type. Methods: Randomly selected from January 2013 to December 2015 in our hospital 96 cases of cervical spondylosis patients treated in our hospital from January 2013 to December 2015 , the technical way of the operation of these 96 cases of loyalty if they were randomly divided into A, B, C three groups. A group of 32 cases were treated by combined anterior and posterior approach in treatment of cervical myelopathy. B group of 32 cases patients with anterior cervical corpectomy decompression and bone graft fusion internal fixation surgery to treat cervical vertebra disease, $\mathrm{C}$ treatment group of 32 patients used conventional posterior decompression and fusion surgery for lateral mass screw fixation. Postoperative follow-up, the three groups of X-ray intervertebral stability and fusion, neurological function JOA score and clinical efficacy of the effective date were compared. Results: three group of patients after half a year, the JOA scores were improved, the patients of the A group after a year of score(16.3 \pm 1.83$)$, the scores of B and $\mathrm{C}$ two groups were respectively $(15.7 \pm 1.15) 、(15.59 \pm 1.21)$, there was statistically differences between the three groups $(\mathrm{P}<0.05)$. After one year's follow-up, the bone graft and internal fixation material had no loosening, displacement and subsidence, the fusion rate of A group reached $90.6 \%$, the fusion rate of B group was $53.1 \%$, the fusion rate of $\mathrm{C}$ group was $56.25 \%$, and the difference was statistically significant $(\mathrm{P}<0.05)$. For clinical effectiveness is divided into apparent, effective, fair, invalid. the effective rate of group A was $87.5 \%$, and the effective rate was $50 \%$ in group $\mathrm{B}$, the difference was statistically significant $(\mathrm{P}<0.05)$. Conclusion: the treatment effect of A group was better than that of $\mathrm{B}$ and $\mathrm{C}$ two groups before and after operation, and the effect was significant.

Key words: anterior and posterior surgery combined therapy; spinal cord type of cervical spondylosis; clinical efficacy; spinal nerve function

\section{Material and methods}

Copyright (C) 2017 JIANG Yan-zhou, et.al.

doi: http://dx.doi.org/10.18686/aem.v6.84

This is an open-access article distributed under the terms of the Creative Commons Attribution Unported License

(http://creativecommons.org/licenses/by-nc/4.0/), which permits unrestricted use, distribution, and reproduction in any medium, provided the original work is properly cited. 


\subsection{General information}

Randomly selected from January 2013 to December 2015 in our hospital 96 cases of patients with cervical spinal cord, these 96 patients according to the different methods of operation were randomly divided into group A (anterior combined therapy surgery) and group B(anterior cervical corpectomy decompression and interbody fusion and internal fixation)and group $\mathrm{C}$ (posterior interbody fusion and lateral mass screw fixation treatment). All patients were diagnosed by clinical diagnosis, CT、MRI and other imaging examinations for the diagnosis of the disease, and other diseases were excluded. These 96 patients 58 males, 38 females, male aged 35-67years old, the average age of

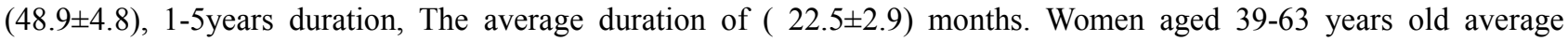

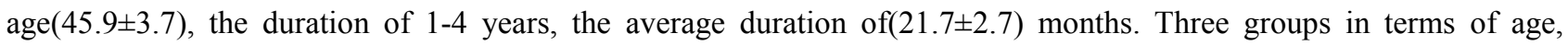
gender and duration of the difference can participation in the contrast $(\mathrm{P}>0.05)$, does not have comparability.

\subsubsection{Symptoms and signs}

cervical spondylosis patients with local pain is a common symptom, which is characterized by neck pain, weakness 、 numbness weakness of the upper limbs, hand fine movement of a sexual disorder. The front of the spinal cord compression performance for the movement dysfunction, lateral disorder manifested as sensory disturbance. The lower limb of the spinal cord after compression is lower than the upper limb. First performance for the lower limb, and gradually develop into muscle tension increased, the late spastic paralysis can occur. Patients can have Hoffman, Babinski positive sign, and appear " feeling separation barrier phenomenon.

\subsubsection{Imaging manifestation}

all patients were examined by X-ray, CT and MRI before admission. The X-ray showed anterior cervical lordosis disappears, and gap, vertebral body bone hyperplasia. CT imaging showed spinal stenosis, calcification of intervertebral disc, protrusion of lumbar intervertebral disc, MRI imaging showed spinal degeneration.

\subsection{Research methods}

all patients were admitted to the hospital research for roution inspection and preoperative examinations, such as blood, urine routine liver and kindney function, early used of antibiotics to prevent infection. In the operation to ensure the patient's breathing unobstructed.

A group of patients with combined anterior and posterior treatment of cervical spondylosis methods: patients in the supine position, on the right side of the neck for transverse incision, the vertebral body exposed, confirming lesion vertebral, intervertebral space in the vertical axis core drilling, scraping the vertebral bone hyperplasia and residual tissue, adjusted the bone column the size to the right, AO plate, fixed by screws. Patients would be adjusted to the prone position. In the middle of the back of the neck incision, skin incision, the paraspinal muscle at the spinous process root separation, drilling, the lamina bone cut off and placed within the plate axis, used the line through hole and shaft and fixed the spinous process, and the surface covered with fat, in the negative pressure drainage, finally wound suture can, patients were given antibiotics, saline, bleeding and other supportive treatment, at 1-year follow-up.

B group of patients before surgical treatment of cervical spondylosis methods:

patient supine, on the right side of the neck shaped transverse incision, the skin incision, the need for reducing vertebral segments exposed, curettage vertebral bone hyperplasia tissue and remaining. After the decompression was completed, the drill core was vertically driven into the intervertebral space, and the titanium plate was fixed with proper size. Patients also gived support to the treatment.

Patients with cervical spondylosis in group $\mathrm{C}$ were treated by routine posterior approach. Specific operations were as follows. The patient was in a prone position. After making a median incision in the neck of the patient, the doctor need 
to make separation of paraspinal muscles, reveal bilateral lamina, spinous process at the end of a long short. Then punch in the spinous process of the root, placing the plate as the pivot, putting it with the door-hinge at the root of the spine in ten silk thread, and covering the gelatin sponge on the surface. Lastly, doctor should place drainage and suture the wound for the patient. All patients were given supportive treatment.

\subsection{Research index}

The three aspects of the experiment results of the three groups of patients about the clinical treatment effect, X-ray cervical spine fusion and spinal nerve function were compared to assess the situation. The spinal nerve function and clinical efficacy were measured by the JOA-17 method of the Japanese Department of orthopedics which is divided into four grades ${ }^{1}$. Score improvement rate of post operation is more than seventy-five percent was a significant effect. Between fifty percent and seventy-five percent was effective. Between twenty-five percent and fifty percent was general. Below twenty-five was ineffective. Using the X-ray to inspect vertebral fixation and fusion. The situation without loosening, displacement or subsidence was valid, but was invalid.

1.4 Statistical analysis: processed by SPSS21.0 system software those data which is using $\mathrm{x}^{2}$ to test count data and using $t$ as inspection standard. Only when the $\mathrm{P}$ value was less than 0.05 , the difference was statistically significant.

\section{Result}

(1) The total effective rate is the sum of markedly effective number and effective number divided by the total number of the group. It was found that the method of treatment is especially indicated in patients with a clinical rate is $87.5 \%$ of $\mathrm{A}$ group was much higher than that of group $\mathrm{B}$ about $50 \%$ and $56.25 \%$ in group $\mathrm{C}, \mathrm{P}<0.05$. (Table 1)

Table 1 Comparison of clinical efficacy between the three groups

\begin{tabular}{lllllll}
\hline group & Cases(n) & Markedly effective & Effective & general & invalid & Effective rate \\
\hline A & 32 & 13 & 15 & 4 & 0 & $87.5 \%$ \\
B & 32 & 1 & 15 & 14 & 2 & $50.0 \%$ \\
C & 32 & 2 & 16 & 11 & 3 & $56.2 \%$ \\
\hline
\end{tabular}

(2) Compared with before treatment, the three groups of patients after surgical treatment, the JOA score are all significantly improved. The JOA score of three groups of patients are statistically analyzed and followed up for six months. The preoperative scores and postoperative year scores of patients in group A are(7.3 \pm 1.7$)$ and (16.3 \pm 1.83$)$. The scores are (7.18 \pm 1.4 )and (15.7 \pm 1.15$)$ in group B. C group's scores were (6.89 \pm 1.21$)$ and(15.59 \pm 1.21$)$. Patients in group A are superior to the other two groups in the recovery of neurological function, $\mathrm{P}<0.05$. (Table 2)

Table 2 Comparison of the score of JOA between the three groups

\begin{tabular}{lccccc}
\hline group & cases & preoperative & \multicolumn{1}{l}{$\begin{array}{l}\text { Six months after } \\
\text { surgery }\end{array}$} & $\begin{array}{l}\text { One year after } \\
\text { surgery }\end{array}$ \\
\hline A & 32 & $7.3 \pm 1.7$ & $15.42 \pm 1.38$ & $16.3 \pm 1.83$ \\
B & 32 & $7.18 \pm 1.4$ & $14.13 \pm 1.18$ & $15.7 \pm 1.15$ \\
C & 32 & $6.89 \pm 1.21$ & $14.24 \pm 1.42$ & $15.59 \pm 1.21$ \\
\hline
\end{tabular}

(3) The X-ray manifestations of the three groups are compared. The situation without loosening, displacement or subsidence is valid. 18 cases had treatment effect, accounting for $90.6 \%$ in group A. The group B had 17 cases which have treatment effect, accounting for $53.1 \%$. There are 18 cases which have treatment effect, accoun-ting for $56.25 \%$. Patients in group $\mathrm{A}$ are superior to the other two groups in the effect of imaging examination, $\mathrm{P}<0.05$. (Table 3 ) 
Table 3 Comparison of the effect of imaging examination between the three groups

\begin{tabular}{lllllc}
\hline group & cases & effective & general & invalid & Effective rate \\
\hline A & 32 & 29 & 3 & 0 & $90.6 \%$ \\
B & 32 & 17 & 5 & 4 & $53.1 \%$ \\
C & 32 & 18 & 9 & 5 & $56.25 \%$ \\
\hline
\end{tabular}

\section{Conclusion}

Cervical spondylotic myelopathy not only have neck pain and limit symptoms, but also have other symptoms including intermittent claudication, leg pain and bowel dysfunction. And cervical spondylotic myelopathy is the most harmful one $^{2}$. At present the clinical use of simple anterior or posterior surgical treatment is not ideal of long-term effect ${ }^{3}$. The combination of anterior and posterior cervical spine therapy can not only reduce the pressure, but also can reconstruct the structure of the cervical spine by bone grafting and fixation which method had achieved good clinical results. In this experiment, we have achieved satisfactory results in the treatment of cervical spondylotic myelopathy with the combination of anterior and posterior.

Cervical spondylosis patients using surgical treatment is to increase the stability of the cervical segment and to improve the overall life quality of patients. The experiment proves that the surgical treatment of the combination of anterior and posterior can relieve the pressure of the front and rear of the cervical which is conductive to the recovery of the function of the spinal cord and spinal stability. The treatment of combined surgery can improve the clinical curative effect of patients and shorten the recovery time. While reducing the pressure on the life, the patients can cost little money on the medical treatment.

Though the comparison of the data of the three groups of patients, patients in group A were superior to the other two groups in the total effective rate of clinical treatment. And patients'result of spinal nerve function score and cervical fusion were better than the other two groups. It is confirmed the therapeutic effect of this surgical approach which is one of the best options. The combined operation method has good clinical effect in the treatment of cervical spondylosis of spinal cord, and it is worth to be popularized in clinical application.

\section{References}

1. Zhang Yilong, Zhou Feifei, Sun Yu. Changes of JOA scores in the near and midterm after surgical treatment of cervical spondylosis myelopathy[J]. Chinese Journal of spinal cord, 2015, 25(1): 13-17.

2. Cui Mingyu. Comparative study on the therapeutic effects of different surgical approaches for cervical spondylosis myelopathy[J]. China Medical Herald, 2012, 09(1):

32-34.

3. Yang Bo, Fan Li, Huang Jing. Anterior and posterior combined surgery for the treatment of anterior and posterior spinal cord compression[J]. Armed police medicine, 2012, 23, (2): 105-107. 